\title{
Localization and Analysis of Critical Areas in Urban Scenarios
}

\author{
Alberto Broggi, Pietro Cerri, Stefano Ghidoni, Paolo Grisleri \\ VisLab - Dipartimento di Ingegneria dell'Informazione \\ Università degli Studi di Parma, ITALY \\ http://vislab.it \\ \{broggi,cerri,ghidoni,grisleri\}@vislab.it
}

\author{
Ho Gi Jung \\ Central R\&D Center \\ MANDO Corporation \\ Yongin-Si 446-901, Korea \\ hgjung@mando. com
}

\begin{abstract}
This paper presents an application of a pedestrian detection system aimed at localizing potentially dangerous situations in specific urban scenarios. The approach used in this work differs from the one implemented in traditional pedestrian detection systems, which are designed to localize all pedestrians appearing in the area in front of the vehicle. This application first locates critical areas in the urban environment, and then it searches for pedestrians in these areas only.

The environment is reconstructed with a standard laser scanner system, while the following check for the presence of pedestrians is performed thanks to the fusion with a vision system.

The great advantages of such an approach are that pedestrian recognition is performed on a very limited image area -therefore boosting its timing performance- and no assessment on the danger level is finally required before providing the result to either the driver or an on-board computer for automatic manoeuvres.
\end{abstract}

\section{INTRODUCTION}

Existing pedestrian detection systems are based on the search for pedestrians in the whole area in front of the vehicle. Candidates are located using pedestrian characteristics [1] such as shape, symmetry, texture, motion, periodicity of human legs motion. When fusion between different sensing technologies is used, being it high-level [2], [3] or low-level [4], each sensor searches for pedestrian-specific features.

In this paper we address a system for the detection of pedestrians based on a new approach. It is designed to work in a particularly challenging urban scenario, in which traditional pedestrian detection approaches would yield nonoptimal results. Moreover, instead of searching for pedestrians in a large area in front of the vehicle and in a generic urban situation, the system presented in this work focuses on a specific scenario in which not only the detection of a pedestrian is of basic importance, but the danger of the situation can be clearly assessed as well. In fact, in an automotive system, a correct detection is just the first phase of a successful product: the localization of a traffic sign/traffic light/obstacle or pedestrian with no corresponding indication on its position with respect to the vehicle and the environment provides very little information, which makes the detection system incomplete. As an example, a pedestrian detection system able to correctly localize all the pedestrians present in the scene provides a huge amount of information, that still needs filtering in order to be useful to either the driver or the on-board computer for automatic manoeuvres. A possible filter may be implemented by fusing information coming from other systems, such as lane detection or other situation analysis engines, and CAN data: a pedestrian exactly in front of the vehicle may or may not be considered as dangerous depending on the surrounding environment as shown in figure 1 .

In this project, VisLab approached the problem in the opposite way: instead of detecting all possible candidates and filter them out after the analysis of the environment, we first assess the scenario, and then search for possible pedestrians in specific positions in that particular scenario. In this way all pedestrians that will be detected are possible threats and no further filtering is needed (a part from a validation and a possible final tracking step). The scenarios that are here considered refer to the very common urban situations in which the presence of a pedestrian poses a serious problem that could be mitigated by an early detection. In particular, when vehicles are moving on an urban road, the only threat that a pedestrian may pose -therefore requiring a successful detection- is a road crossing. Conversely stopped vehicles on the road or on the road sides create a completely new set of scenarios, in which the presence of a pedestrian might be absolutely dangerous.

The underlying idea is to localize stopped vehicles and then search for pedestrians in the close proximity of these vehicles or in the areas hidden by them. These stopped vehicles, whose edges will trigger the search for pedestrians, can be parked cars on the road edge, vehicles temporarily stopped on the road, or vehicles queued in a line in front of a traffic light, zebra crossing, or simply jammed.

The first row of figure 2 shows some examples of situations in which the visibility of a crossing pedestrian is occluded by stopped vehicles. The second row of figure 2 highlights, for each situation, the areas on which the system will perform a check for the presence of a possible pedestrian. A straightforward extension -which is not currently investigated in this project- is to locate large static structures (such as buildings or guard-rails) and treat them as stopped vehicles as in the above description.

In other words, this paper focuses on the detection of pedestrians popping out behind stopped vehicles; pedestrians that are clearly visible in the frontal area of the vehicle can also be detected, but this function -common to other systems 


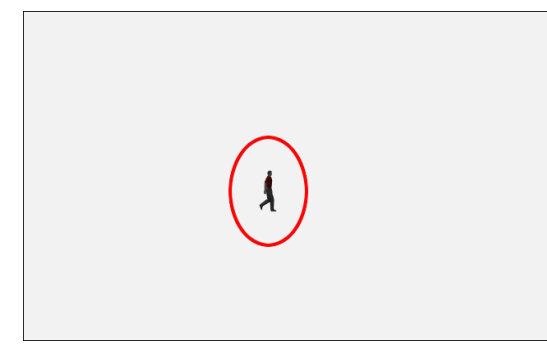

(a)

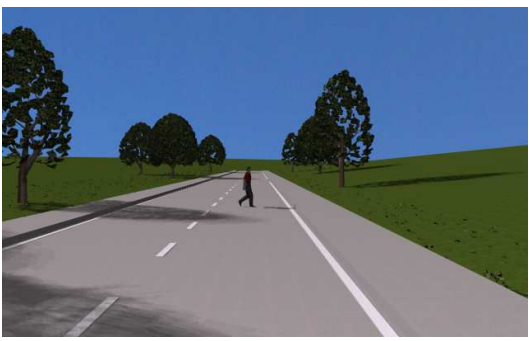

(b)

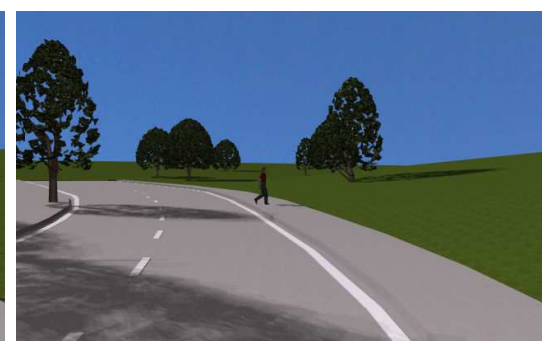

(c)

Fig. 1. When a pedestrian is localized but no reference with respect to the environment is provided like in (a), the detector is not able to assess the danger level. When fusing environmental information, the very same pedestrian may become a threat $(b)$, or may be in a safe position $(c)$.

[5] [6]- is out of the scope of this article.

The idea of focussing on a specific scene is not new to pedestrian detection systems: in 2002 Franke and Heinrich [7] developed a module able to detect balls (which are usually a strong signature of the presence of a child).

It is known [8] that parked vehicles, blocking the visibility of pedestrians, are one of the main causes of accidents: in residential areas, in fact, parking spaces should be arranged diagonally. However, as shown in figure 2, there are situations in which vehicles stop temporarily on the road and their position cannot be strategically organized and carefully managed as in the case of parking lots. Although some of these situations refer to specific urban areas (i.e. zebra crossings and bus stops) that could be specifically enhanced by intelligent infrastructures aimed at warning oncoming vehicles, other situations can happen in any spot of the road network, making the installation of intelligent infrastructures impractical.

The main characteristic required by such a system is the capability to:

- detect pedestrians quickly, given the short working range and the particular danger of an imminent collision;

- detect pedestrians as soon as they appear, therefore even when they are still partly occluded;

- limit the search to given areas, determined by a quick pre-processing.

The system presented in this work addresses a specific application of a pedestrian detection system, which is being currently developed and tested by VisLab in Parma on a Hyundai Grandeur prototype vehicle; MANDO is using a replica of this system which is being tested in Korea.

In the next section the test vehicle setup is presented, then in section III a description of the risky area detection can be found. In section IV considerations about vision fusion are discussed, and in section V some final remarks are exposed.

\section{The Test Vehicle}

\section{A. Sensing Technologies}

Being designed to address an urban scenario in which the prototype vehicle is running close to stopped vehicles, a limitation on vehicle speed and detection range is straightforward. Low-to-medium vehicle speeds up to $50 \mathrm{~km} / \mathrm{h}$ and a detection range of about $40 \mathrm{~m}$ can be considered as a safe choice.
Environmental sensing requires stopped vehicles to be detected; a laser based solution is sufficiently strong to localize large obstacles like vehicles and classify them thanks to their shape. To overcome the lack of speed information associated to laser scans, we selected a particular laser scanner with the specific characteristic of providing interlaced data. It groups a number of interlaced scans together to form a single higherresolution scan. The analysis of this interlaced data coupled to vehicle CAN data allows to estimate obstacles speed and therefore to localize vehicles with null speeds.

The best technology to check for the presence of a pedestrian in a given set of specific spots is vision. Monocular vision is sufficient since no 3D reconstruction is needed and an estimation of the distance is already available. Although the system is now being tested in daylight conditions, the use of a NIR camera and proper illumination allows to extend its operational range to the night.

\section{B. Sensor Selection}

The camera has sensitivity both in the visible and NIR spectra. Additional head lamps are mounted in front of the vehicle. A NIR LED headlight with an aperture of about $25^{\circ}$ degrees is mounted in front of the radiator, and the high beams are customized by SL to block the visible light and illuminate in the NIR spectrum only.

The laser scanner is a SICK LMS 211-30206. The detection capabilities (scanning angle of $100^{\circ}$ degrees, minimum angular resolution of $0.25^{\circ}$ degrees, up to 80 meters range, fog correction) are suitable for our goal.

The laser scanner is capable of $1^{\circ}$ degree resolution, but thanks to 4 subsequent rotations, using an interlacing system, and a phase-gap of 0.25 degrees, it is possible to decrease the final granularity to $0.25^{\circ}$ degrees. Every rotation takes $13.32 \mathrm{~ms}$, so, in $53.28 \mathrm{~ms}$ four rotations are performed. The time difference between the pulses measurement is not negligible; moreover when the vehicle is moving, the shift between the position of the laser scanner when the first pulse is measured, and the position when the last pulse is measured is appreciable: the interlacing system of the laser scanner make this problem even more evident.

\section{Vehicle Setup}

A compact $\mathrm{PC}$ is mounted in the boot, while a laser scanner and two different NIR head lamps are located in the front 

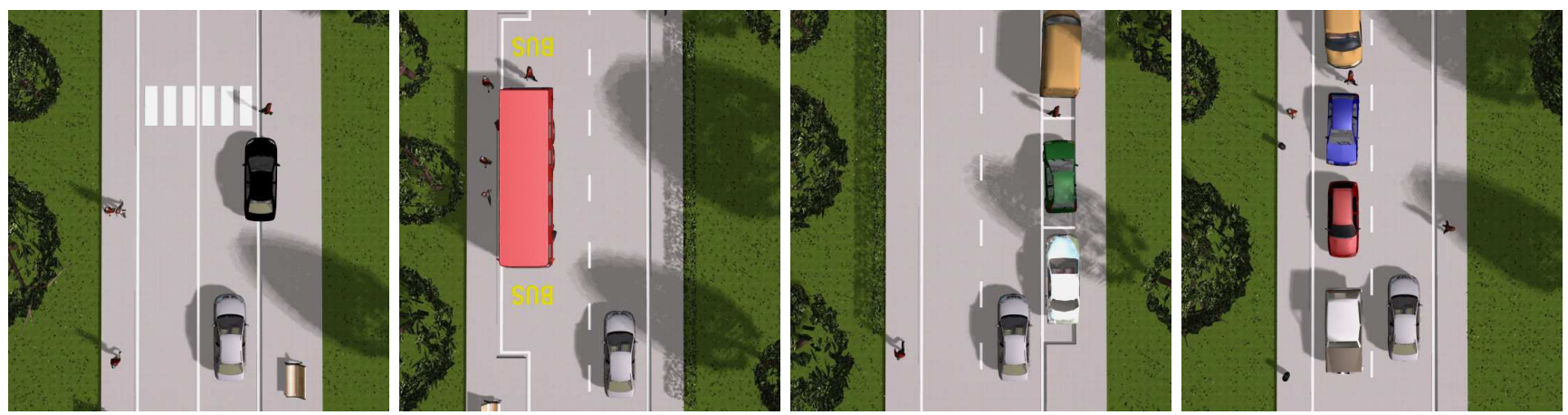

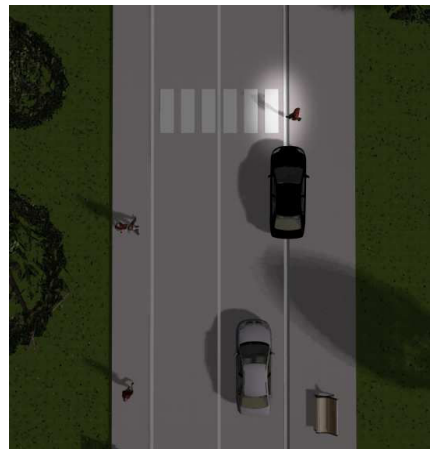

(a)

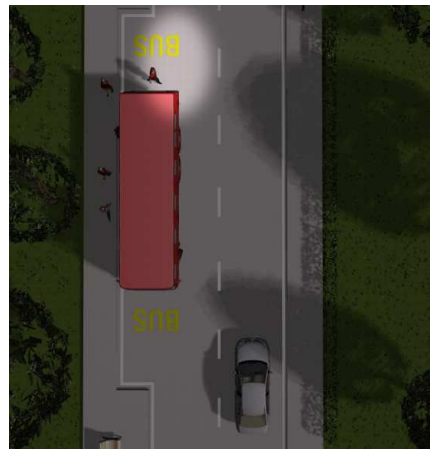

(b)

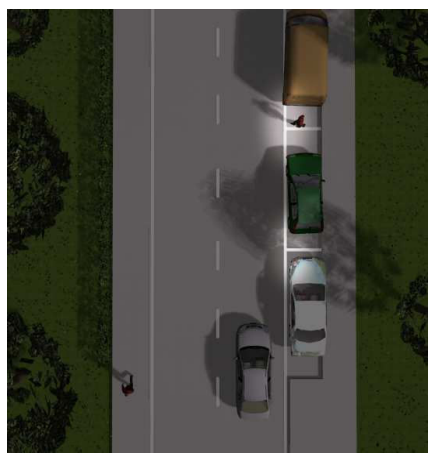

(c)

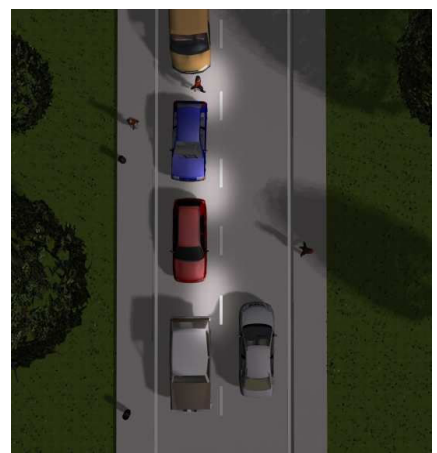

(d)

Fig. 2. Situations considered in this work (first row) and areas of interest considered for the detection of a possible pedestrian (second row): (a) a crossing pedestrian is hidden by a parked vehicle; $(b)$ a pedestrian is crossing the road behind a stopped bus; $(c)$ a pedestrian is popping out in between two parked vehicles; $(d)$ a pedestrian is crossing the road between two vehicles stopped on the other side of the road. It is important to note that situations $(a)$ and $(b)$ refer to specific and localized urban areas (zebra crossings and bus stops); situations $(c)$ and $(d)$ may happen in any portion of the road network.

bumper. The NIR camera is placed inside the cabin near the rear-view mirror, as shown in figure 3.

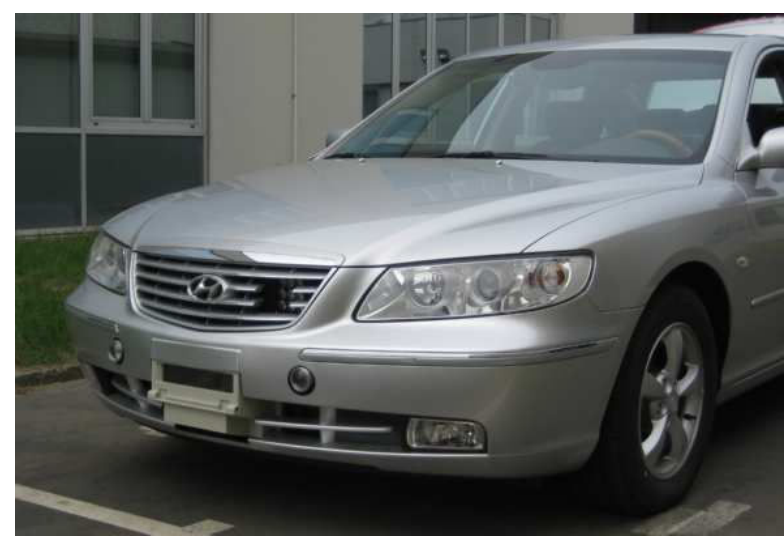

Fig. 3. The Hyundai Grandeur test vehicle equipped in Parma.

\section{FocUS OF ATTENTION}

Two different laser data classifications are developed in order to make the algorithm robust. Both methods are based on pulses clustering into straight lines, but, while the first one considers only the last scan to classify obstacles, the second one uses a fixed number of previous scans to verify the previous position of obstacles and therefore estimate their speed.

\section{A. Data correction}

As already explained in section II, shifts between subsequent laser scanner measurements can be appreciable: this feature may cause clustering or classification problems, because obstacles shape may appear distorted.

Using ego motion data provided on the $\mathrm{CAN}$, it is possible to compute the roto-translation of the vehicle and correct the position measured for each pulse.

Figure 4 shows raw laser data and corrected data referring to non-moving obstacles. The four rotations that create a scan are clearly visible before the correction, while the obstacle appears as a single line after the correction.

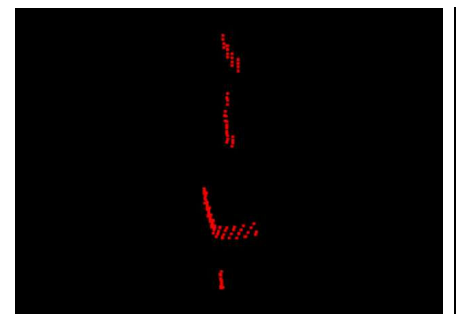

a

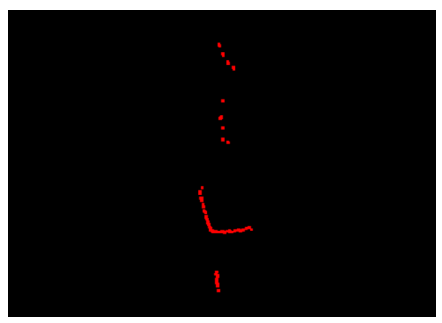

b
Fig. 4. Laser data: (a) without correction and (b) with correction.

Pulses echoed by moving obstacles can not be reduced to a single line, since the position of the obstacle changes during the scan: moving obstacles are identified by four parallel lines 
after the data correction. This information can become very useful for obstacle classification.

\section{B. Data clustering}

Connecting subsequent pulses, while discarding information about the rotation at which the pulse belongs to, works for all situations except for moving obstacles. Better results can be obtained by connecting only the pulses that belong to the same rotation: moving obstacles are then identified by four different and parallel clusters. The pulses are clustered as chains of segments. The points that can not be joined into any chain of the same rotation, are then tested for merging with points of other rotations considering proximity only. The points that can not be connected to any other point or are on the border of laser scanner range (about 80 meters) are permanently discarded.

\section{Segment merging}

Up to now pulses are joined in a chain of segments without any other information. Adjacent segments with approximately the same orientation can be merged into a longer segment, preserving the obstacle shape, but reducing the complexity of the data structure and the details of the representation. Each chain is then segmented into a polyline.

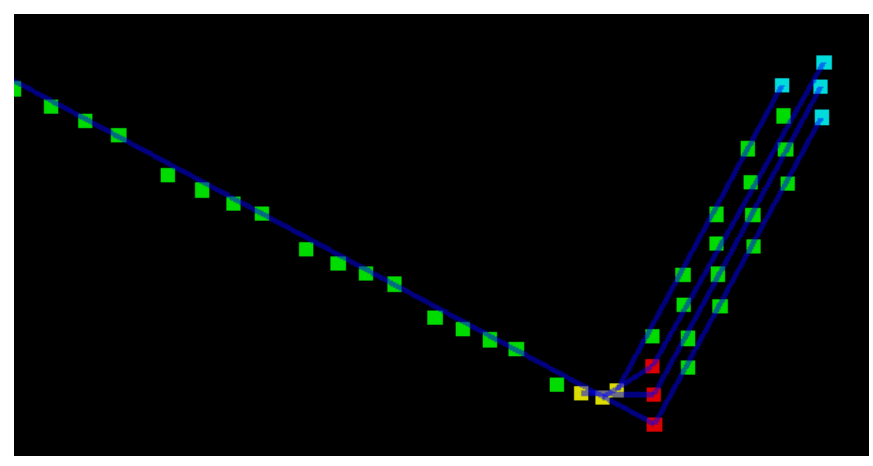

Fig. 5. A moving vehicle: the four parallel lines correspond to its bumper, while the single line corresponds to its side. Yellow points are polylines start points, red points are points in the middle of polylines, cyan points are polylines end points, green points are points belonging to a line.

Figure 5 shows a moving vehicle: the rear bumper is framed as four parallel lines, while its side, that it is parallel to the the vehicle movement direction, is marked by a single line.

\section{Lines merging}

Every obstacle in the field of view of the laser scanner is identified by four lines, one for each rotation that composes the whole high resolution scan. In the case of static obstacles the four lines are quite perfectly overlapping, thanks to the previous data correction, and can be merged. Conversely in the case of moving obstacles the lines are parallel but not overlapping, therefore static and moving obstacles can be located and correctly classified. Figure 6 shows the steps of the algorithm; figure (c) shows the final result, where all the points are merged into a single line: in order to simplify the following steps, little drift are ignored. This quick and simple, yet very effective, process to identify static obstacles may generate false negatives when the vehicle's pitch is not negligible. Section V will discus this issue.

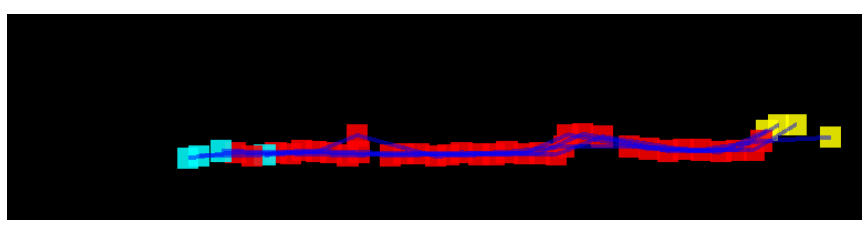

(a)

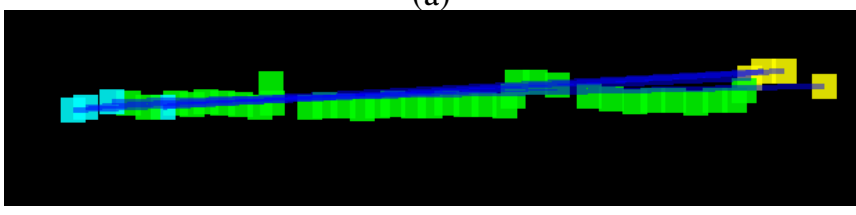

(b)

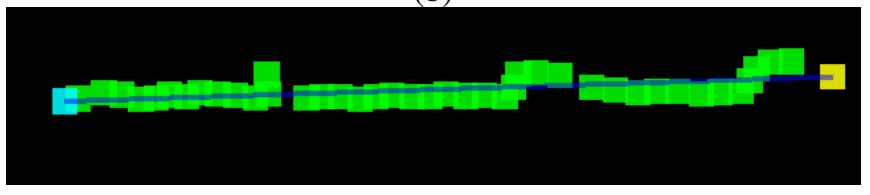

(c)

Fig. 6. The steps of the algorithm: (a) data clustering, $(b)$ approximation using polylines, and $(c)$ lines merging.

\section{E. First obstacle classification}

The obtained polylines can be classified according only to their size and shape. It is possible to divide obstacles into five categories:

- possible pedestrian,

- road border,

- L-shaped obstacle,

- moving obstacle,

- generic obstacle.

Obstacles that can eventually be classified as pedestrians are supposed to have a reduced size, while obstacles that have a large size and are almost parallel to the vehicle, are assumed to be road borders (guard-rails, road infrastructures, ...). A simple and fast method, based on lines orientation, is used to detect L-shaped obstacles. Moving obstacles are already individuated in the previous step of the algorithm. All obstacles that are not yet classified, are tagged as generic obstacles. The results obtained so far are satisfactory as shown in figure 7.

\section{F. Temporal and shape-based correlation}

The classification explained in the previous subsection classify all the tiny obstacles as possible pedestrian. Even if it is possible that all pedestrians are correctly classified, a number of false positives are present. Fixed obstacles along the road (especially parked vehicles) are here used to localize critical areas in front of the vehicle, in order to focus the attention in the immediate proximity of the edges of this risky area, where pedestrians can pop out and become dangerous. 


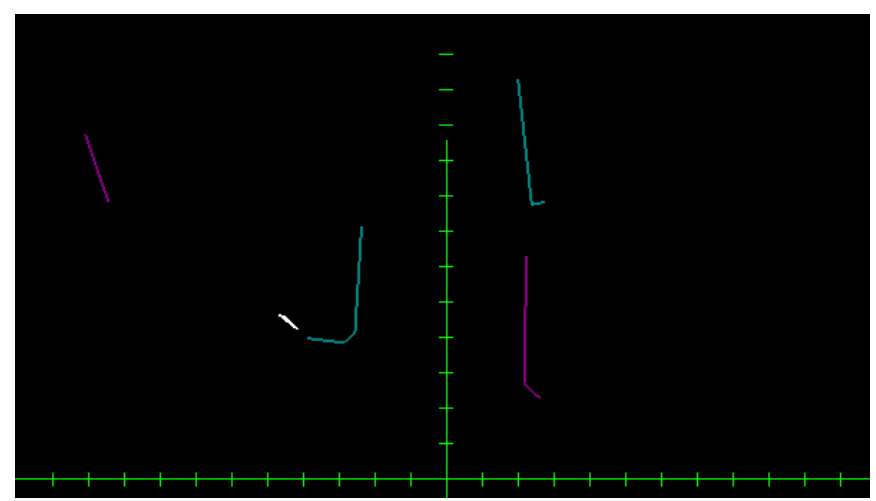

Fig. 7. Obstacle classification: cyan lines refer to L-shaped obstacles, white ones to possible pedestrians, and violet ones to generic obstacles.

Polylines provided by the previous scans are moved according to ego-motion; then the overlap between the current polylines and the previous ones is checked in order to provide a new classification in the following 4 classes: moving obstacle, static obstacle, changing shape obstacle, and new obstacle.

Scan data referring to moving obstacles should have little or no spatial overlap when the time window is large, but unfortunately, the side of a moving vehicle appears as static (high spatial overlap). Anyway, thanks to the previous labelling of L-shaped objects, the bumper and the sides of the same vehicle belong to the same object which therefore inherits the 'moving' label.

Obstacles that are represented by well overlapping scans in the given time window are marked as static, even when the newer scan lines are longer and more accurate than the older ones (due to the vehicle getting closer and closer to the obstacle).

Whenever scanlines are detected which overlap only in some regions and differ in others, the object is classified as a changing shape obstacle. This may happen when some new road player (such as a pedestrian or a vehicle which was previously occluded) comes into the scene: being close to another object, in the laser data it appears as its shape were connected to the shape of its adjacent obstacle. It may also happen when a driver of a stopped vehicle opens the door and gets out of the vehicle.

When there is no correspondence between the current polyline and old ones, the object is classified as a new obstacle.

Moving obstacles are discarded; static obstacles are important to locate the areas of interest in which vision will search for pedestrians; changing shape obstacles are of basic importance since they may contain a pedestrian in a very precise region of their shape; and finally new obstacles locate other areas of interest in which vision will have to work.

\section{G. Risky area identification}

All the information obtained in the previous steps can be used to identify the environment (static obstacles and vehicles) and the moving obstacles (pedestrians and vehicles).
Obstacles identified as static by the second classification, and not classified as moving by the former, are used to define the structure of the environment. Also changing shape obstacles are used to built the environment, considering only points that are not changing. Non-moving obstacles in the right and left part of the road in front of the vehicle are used to build the right and left border of the risky area, namely the area that may be reached by the vehicle in the near future, as shown in figure 8 .

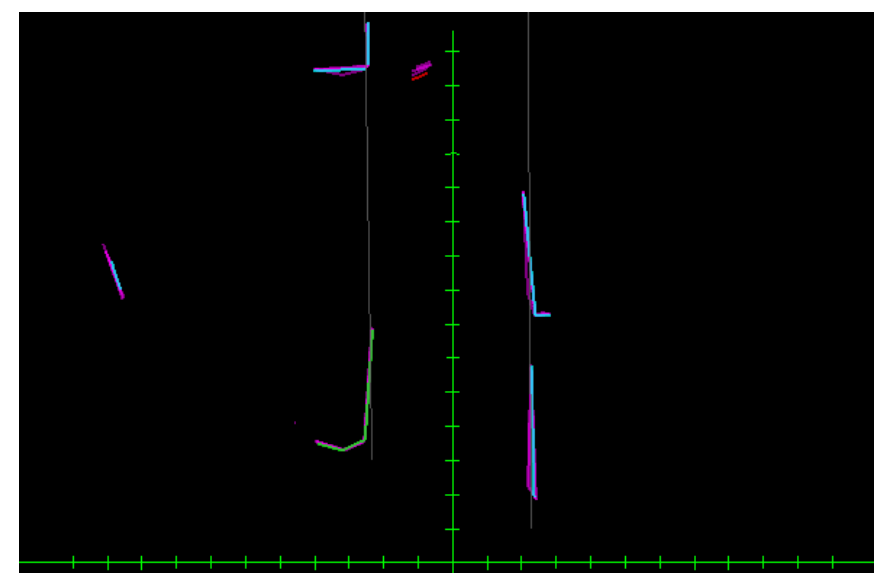

Fig. 8. Obstacle classification and risky area: violet lines are position of obstacles provided by previous scans, red lines are mobile obstacles, cyan lines are static obstacles, green lines are changing shape obstacles, while gray lines represents left and right border of risky area.

Obstacles moving in the risky area are tagged as risky obstacles. Attention is especially focused on the areas between static obstacles. Dangerous pedestrians that need to be detected will appear behind a static obstacle or will modify the shape of a static obstacle.

\section{H. Results of the attentive process}

Thanks to the projection of laser data on images, figure 9 shows a case where a pedestrian is popping out behind a parked vehicle and another pedestrian is walking in the risky area. Both pedestrians are correctly detected. Figure 9 shows a van popping out from a parking and a cyclist riding near the parked vehicles: both dangerous obstacles are correctly detected. The execution time is less than 5 milliseconds using a Pentium $42.8 \mathrm{GHz}$ PC; about 48 milliseconds remain to the vision fusion to keep real-time constraints.

\section{VISION FUSION}

Regions of interest are 2D areas in world coordinates; their corresponding areas in the image are then located thanks to camera calibration and perspective mapping. Indeed camera calibration, and more generally system calibration, plays a basic role in a fusion system and needs to be tackled.

Vehicle's pitch during motion may change camera orientation with respect to the world, but -most importantlyit also causes the laserscanner to scan the scene using a different orientation. For on-road applications this is generally considered a limited issue for short distance sensing, 


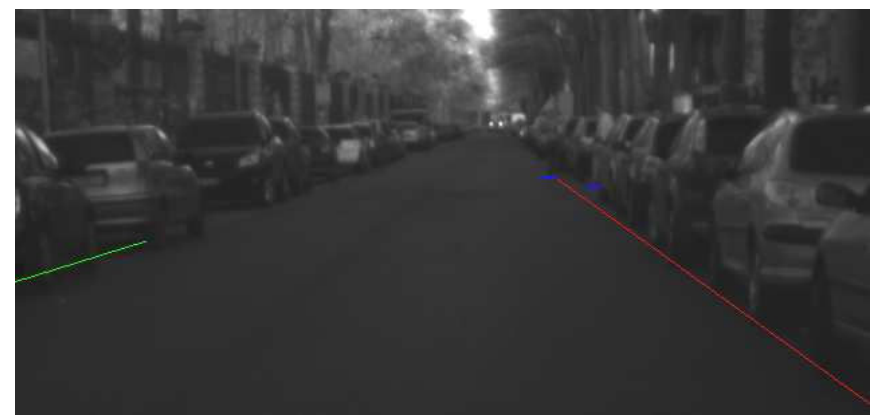

Fig. 9. Dangerous pedestrians correctly identified.

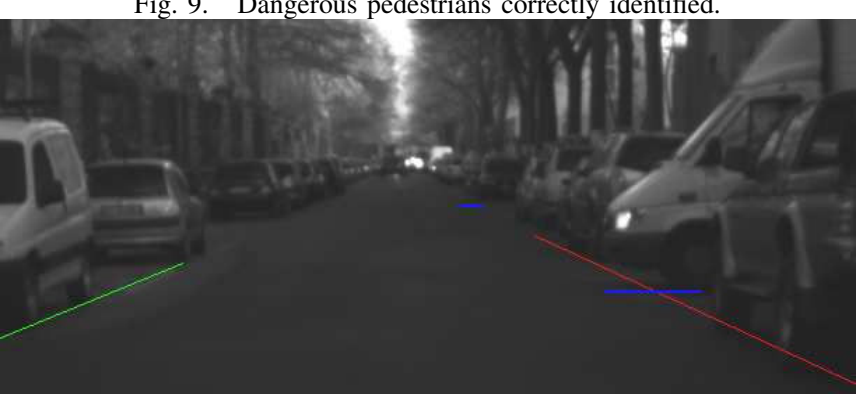

Fig. 10. Dangerous obstacles correctly identified.

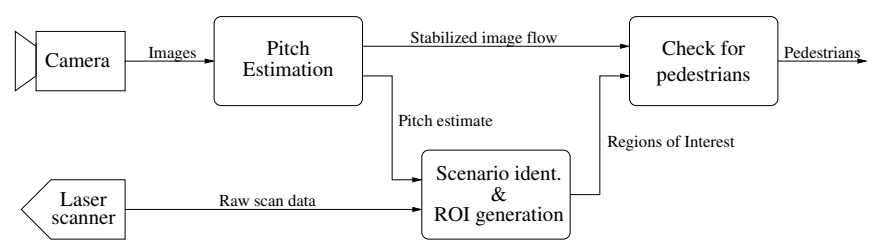

Fig. 11. The scheme used to fuse vision and laserscanner data.

and this is even less important when obstacles are vertical. Conversely, in case of appreciable vehicle's pitch angles especially negative angles that point the laserscanner towards the ground- the laserscanner's scanning plane may intersect the ground and therefore yield non significant results.

Thanks to the fact that the two sensors are fixed to the vehicle's frame, they move accordingly and a single compensation may be sufficient. Vision, besides being used to locate pedestrians' features in the regions of interest, is also used to estimate vehicle's pitch [9]. An additional accelerometer is now being used to check pitch measurement results provided by vision. Instantaneous vehicle's pitch is fed to the laserscanner processing (in order to inhibit the generation of wrong results during vehicle pitching), and is also used to stabilize the image stream, as shown in figure 11.

Once the areas of attention are located, the search for pedestrians is triggered using AdaBoost classifier [10]. The identified areas are resampled to a fixed size $(50 \times 20)$ and all the pixel are used as input. AdaBoost was trained using areas individuated by the previous steps of the algorithm and manually marked as pedestrian or not.

\section{Conclusions}

This paper presented a new scheme to increase safety and possibly avoid collisions with vulnerable road users. Instead of implementing a generic pedestrian detection system followed by both a validation step and the assessment of the danger level, this work proposes an innovative approach. Whenever a specific environment structure is detected (i.e. stopped vehicles that may hide pedestrians), the possible presence of pedestrians is checked in specific areas.

Not only this solution approaches the problem from a different perspective with respect to traditional implementations [1], but it also focuses on a particularly critical environment, typical of urban accidents. Besides directly providing detections of dangerous situations, it also boosts timing performance, since the computationally intensive part -vision-based pedestrian recognition- is performed only on limited portions of the image. Speed-up due to the critical area localization step can be measured as the difference between the number of possible pedestrians generated by a classical system (that considers only size to locate pedestrians) and the ones generated by this method: several tests in urban scenarios show that the proposed method provide on average less than a quarter of possible pedestrians than classical system.

The next research step is the extension of the image processing part to include tracking. The final step will be the integration of other critical urban scenarios.

\section{REFERENCES}

[1] T. Gandhi and M. M. Trivedi, "Pedestrian Protection Systems: Issues, Survey, and Challenges," IEEE Trans. on Intelligent Transportation Systems, vol. 8, no. 3, pp. 413-430, Sep. 2007.

[2] M. Szarvas, U. Sakai, and J. Ogata, "Real-time Pedestrian Detection Using LIDAR and Convolutional Neural Networks," in Procs. IEEE Intelligent Vehicles Symp. 2006, Tokyo, Japan, Jun. 2006, pp. 213-218.

[3] S. Milch AND M. Behrens, "Pedestrian Detection with Radar and Compuer Vision," http://www.smartmicrowave-sensors.de/Pedestrian_Detection.pdf.

[4] A. Broggi, A. Cappalunga, C. Caraffi, S. Cattani, S. Ghidoni, P. Grisleri, P. P. Porta, M. Posterli, P. Zani, and J. Beck, "The passive sensing suite of the TerraMax autonomous vehicle," in Procs. IEEE Intelligent Vehicles Symposium 2008, Eindhoven, Netherlands, Jun. 2008, in Press.

[5] C. Premebida, G. Monteiro, U. Nunes, and P. Peixoto, "A Lidar and Vision-based Approach for Pedestrian and Vehicle Detection and Tracking," in Procs. IEEE Intl. Conf. on Intelligent Transportation Systems 2007, Seattle, WA, Sep.-Oct. 2007, pp. 1044-1049.

[6] J. P. Hwang, S. E. Cho, K. J. Ryu, S. Park, and E. Kim, "MultiClassifier Based LIDAR and Camera Fusion," in Procs. IEEE Intl. Conf. on Intelligent Transportation Systems 2007, Seattle, WA, Sep.Oct. 2007, pp. 467-472.

[7] U. Franke and S. Heinrich, "Fast obstacle detection for urban traffic situations," IEEE Trans. on Intelligent Transportation Systems, vol. 03, no. 3, pp. 173-181, Sep. 2002.

[8] R. A. Retting, S. A. Ferguson, and A. T. McCartt, "A review of evidence- based traffic engineering measures designed to reduce pedestrian's motor vehicle crashes," Amer. J. Public Health, vol. 93, no. 9, pp. 1456-1463, Sep. 2003.

[9] L. Bombini, P. Cerri, P. Grisleri, S. Scaffardi, and P. Zani, "An Evaluation of Monocular Image Stabilization Algorithms for Automotive Applications," in Procs. IEEE Intl. Conf. on Intelligent Transportation Systems 2006, Toronto, Canada, Sep. 2006, pp. 1562-1567.

[10] S. Munder and D. M. Gavrila, "An Experimental Study on Pedestrian Classification," IEEE Trans. on Pattern Analysis and Machine Intelligence, vol. 28, no. 11, pp. 1863-1868, Nov. 2006. 